\title{
Microhematuria as an indicator of significant abdominal injury
}

Authors: Lea Ohana-Sarna-Cahan MD (1), Yonatan Levin (5), Itai Gross MD (2), Ayal Hassidim MD(4), Jonathan Benjamin Yuval MD(3), Amit Hess MD (2), Miklosh Bala MD (3), Saar Hashavya MD( ${ }^{(2)}$

\section{Affiliations:}

(1) Department of Pediatrics, Hadassah-Hebrew University Medical Center, Jerusalem

(2) Department of Pediatric Emergency Medicine, Hadassah-Hebrew University Medical Center, Jerusalem

(3) Department of General Surgery, Hadassah-Hebrew University Medical Center, Jerusalem

(4) Department of Plastic and Reconstructive Surgery, Hadassah-Hebrew University Medical Center

(5) Faculty of Medicine, Hadassah-Hebrew University Medical Center

Results: The charts of 655 children were reviewed. Microhematuria was found in 100 children of whom $49(49 \%), 28(28 \%)$ and $23(23 \%)$ had small, moderate and large amounts of hematuria respectively. Forty-one of the children who had microhematuria underwent a computed tomography (CT) scan. Positive findings were recorded in 16 of these patients (39\%). There was clear association between microhematuria as detected by the urine dipstick and a significant finding on the CT scan $(p=0.002)$. The sensitivity of microhematuria for significant abdominal pathology on imaging was $66.6 \%$ and the specificity was $68.3 \%$ (PPV $=39 \%$, NPV $87.1 \%)$. Microhematuria was associated with increased length of stay (LOS) in the hospital $(p<0.001)$, surgical interventions $(p=0.036)$ and admission to the pediatric intensive care unit $(I C U)(p<0.001)$.

Background \& Objectives: Trauma is one of the leading causes of morbidity and mortality in the pediatric population. In many centers, microhematuria is used as a screening tool for the presence of significant abdominal injury and as an indication for further imaging. Our objective was to evaluate the role of microhematuria by

dipstick as an indicator of significant abdominal injury in children.

Method: A retrospective review of children aged 0-16 years admitted for MVA or a fall from 2007 to 2017, who had urinalyses performed.

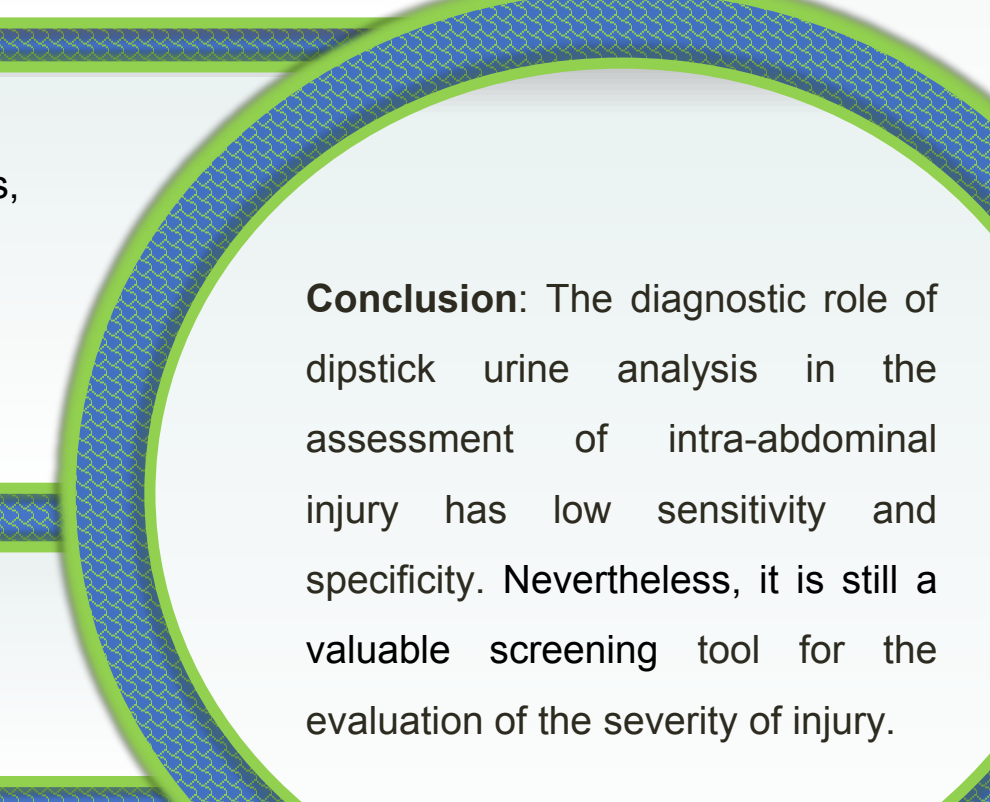

evaluation of the severity of injury.

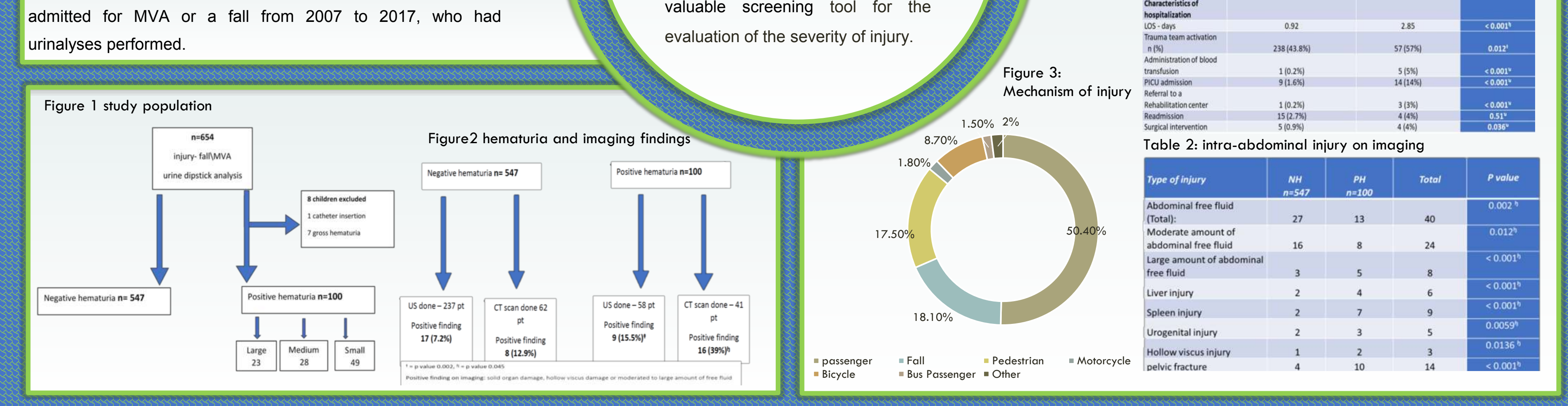

